\title{
INFLUENCIA EDUCATIVA DE LOS PADRES EN UNA VISITA AL MUSEO DE LA CIENCIA: ACTIVIDAD COMPARTIDA ENTRE PADRES E HIJOS FRENTE A UN MÓDULO
}

\author{
BENLLOCH, MONTSE ${ }^{1}$ y WILLIAMS, VILMA N. ${ }^{2}$ \\ ${ }^{1}$ Facultad de Educación. Universidad de Vic. \\ 2 Departamento de Psicología de la Universidad Nacional de la Plata. Argentina.
}

\section{SUMMARY}

This study intends to determine the educational influence of eighty-four parents over their children at a module at Science Museum. The study of this influence, on the one hand, examined a number of behaviours shared during the day at the activity centre known as «The Wave Machine» by analysing attention, disinterest, reading of signs and dialogue. On the other hand, the influence of several demographic characteristics of the population, such as the educational formation and type of parents using the activity centre, are studied. Both variables are analysed using contingency charts and establishing the degree of significance among the categories of each.

The results obtained demonstrate significant differences in the area of communication. The parents with a university education speak more with their children as compared to those with another type of educational formation.

\begin{abstract}
SÍNTESIS
Este trabajo se propone conocer la influencia educativa de 84 progenitores sobre sus hijos frente a un módulo del Museo de la Ciencia de Barcelona. Se estudia dicha influencia examinando, por una parte, algunas modalidades de conducta en la actividad compartida entre cada díada frente al expositor «La máquina de las olas»: atención, desinterés, lectura de carteles, diálogo son analizadas. Por otra parte, se estudia la influencia de algunas características demográficas de la población, como son la formación y el género de los progenitores en dicha actividad. Ambas variables son analizadas empleando tablas de contingencia y estableciendo el grado de significación entre las categorías de cada una.
\end{abstract}

Los resultados obtenidos presentan diferencias significativas en la esfera de la comunicación. Los padres y las madres que poseen una formación universitaria dialogan más con sus hijos frente al módulo que aquéllos que poseen otro tipo de formación.

\section{CENTROS DE CIENCIA Y APRENDIZAJE INFORMAL}

El éxito de audiencia que han experimentado los centros de ciencia en los últimos años se debe, probablemente, a que ofrecen espacios interesantes para la comunicación y el aprendizaje aunque su objetivo último sea divulgar de modo divertido y sugerente la cultura científica. En este sentido los museos o centros de ciencia son escenarios de aprendizaje informal (Dierking y Falk, 1994; Wellington, 1990) cuyos entornos multisensoriales son cada vez más apreciados y elegidos libremente por las familias para consumir su tiempo libre. Conocer su influencia educativa requiere tener en cuenta tanto el contexto inherente al propio escenario como el rol fundamental que juega la motivación de los visitantes.

Se entiende por aprendizaje en este contexto, no sólo la adquisición de hechos y conceptos científicos sino también, o más bien, la posibilidad de aplicar las ideas aprendidas en las exposiciones, así como el cambio de 
algunas actitudes, las experiencias novedosas y también las conversaciones e interacciones socialmente mediadas entre los grupos de amigos o entre miembros de las familias que visitan los museos.

Este último punto se hace especialmente relevante en las visitas familiares. La familia ejerce de mediadora para impulsar este tipo de aprendizajes en muchas y variadas situaciones de más larga tradición; sin embargo, en las ciudades donde hay algún centro de ciencia empieza a ser una práctica familiar, relativamente frecuente, visitarlo durante los fines de semana.

A menudo los aprendizajes que tienen lugar en el seno familiar ocurren en el transcurso de la actividad habitual de los adultos y en los contextos naturales, la casa, ir de compras, ir al médico, al parque, entre otros. En estas actividades los jóvenes toman parte en función de sus posibilidades. Muchos estudios sobre este tipo de aprendizajes han puesto de manifiesto que «los procesos sociales e institucionales están estructurados para permitir la adquisición por parte del niño de las destrezas, valores, actitudes y costumbres básicas que definen la conducta adulta apropiada en esa cultura» (Scribner y Cole, 1973, trad. cast. 1982, p. 9). Así mismo, un enfoque sociocultural enfatiza las ventajas para el aprendizaje y desarrollo de las oportunidades educativas que ofrecen muchos escenarios no formales (Gelman et al., 1991; Valsiner, Man-Chi Leung, 1994).

El término aprendizaje aplicado a una actividad no reglada goza de una definición muy amplia y abarcadora. Una de las más empleadas entre los investigadores (Falk y Dierking, 1992) es la de Ausubel, Novak y Henesian «[...] hay aprendizaje significativo si la tarea de aprendizaje puede relacionarse de modo no arbitrario y sustancial (no al pie de la letra) con lo que el alumno ya sabe [....]» (Trad. cast. 1983, p. 37). Sin embargo, a la etiqueta clásica de «aprendizaje significativo» hay que añadir un amplio rango de posibles procesos y resultados, específicos en este caso, de un escenario formal. Entendiendo por «formal» la intención educativa que típicamente dirige el diseño de los módulos en los museos o centros interactivos, esos diseños tratan de favorecer intencionalmente un proceso de aprendizaje. Pero además esos aprendizajes tienen un carácter no institucional en el sentido dado por Scribner y Cole (en un artículo ya clásico 1973/1982); en los museos, los visitantes deciden lo que quieren aprender y son libres de planificar el modo de hacerlo.

Los aprendizajes sostenidos por las familias que visitan los museos presentan, pues, características específicas.

Muchas de las investigaciones sobre familias y aprendizaje (Bulter, Sussman, 1989) parten del supuesto de que la experiencia es posible gracias a los mecanismos de socialización y comunicación que tienen lugar en los diferentes escenarios interactivos. Estos mecanismos se ponen de manifiesto mediante conductas que se adoptan como indicadores de aprendizaje. Algunas de las más mencionadas son entre otras: el tiempo que emplean los adultos observando el módulo, la lectura efectiva de los carteles, su intensidad y la comunicación con los niños.

\section{CARACTERÍSTICAS DEMOGRÁFICAS Y COMPOSICION DE LOS GRUPOS FAMI- LIARES}

En una extensa revisión sobre los visitantes y las visitas a museos y centros de ciencia, Diarking y Falk (1994) señalan que, aun cuando las investigaciones sobre grupos familiares en relación con el conjunto de investigaciones museísticas es bastante escaso, éstas agrupan un amplio abanico de características y conductas de dichos grupos.

Un acuerdo bastante generalizado entre los investigadores (Lakota, 1975; Rosenfeld, 1979, 1981) es que las familias y grupos de adultos difieren en su conducta. En las familias son los adultos quienes seleccionan los recorridos eligiendo dónde detenerse. Muestran a los niños aquello que les parece más interesantes o aquello cuya información les resulta más familiar. En esta misma línea, otros autores (McManus, 1987; Diamond, 1980, 1986) señalan que la conducta de mujeres y hombres difiere. Hay mayor probabilidad de que las mujeres sean más exploratorias cuando van solas que cuando van acompañadas de hombres. Las madres cuando van con niños son más sumisas a los niños que los padres, quienes con mayor probabilidad adoptan el papel de líder.

Algunas de las conductas más observadas entre los miembros de los grupos familiares y que han llamado la atención de los investigadores son la comunicación verbal que se establece entre padres e hijos frente a los expositores, la lectura de carteles o gráficos y la manipulación directa sobre los expositores. Estas conductas han sido estudiadas comparando cómo las ejecutan diferentes miembros de los grupos familiares. Por ejemplo, Diamond $(1980,1986)$, en un estudio realizado en el Lawrence Hall y en el Exploratorium de San Francisco, observa diferentes patrones de interacción y exploración entre padres e hijos. Los niños manipulan más que los padres y éstos leen más que los niños. Así mismo, este autor encuentra diferencias en la conducta interactiva de padres e hijos y madres e hijas.

Cuando un hijo y su padre se acercan a un módulo, éste puede leer, señalar y comentar o preguntar sobre el contenido del mismo. Diamond, sin embargo no observó este tipo de interacción entre madres e hijas.

La manipulación de los expositores y la lectura de los textos o carteles han sido también objeto de examen. Un hallazgo general (Diamond, 1980, 1986) es que muchas familias no leen participativamente las instrucciones del expositor. Prefieren comprender primero mediante la manipulación y, si tienen éxito o siguen interesados, entonces leen.

La atención ha sido ampliamente estudiada. Schouten (1987) establece que la conducta de abandono del museo 
que orienta al visitante hacia la salida puede estar motivada por diferentes factores que dificultan la atención y que suelen ser comunes a muchas exposiciones y museos: uniformidad en el diseño, presentación estática, diseño típico de museo, diferentes niveles de abstracción, presentación dominada por el punto de vista científico, insuficiente conexión con el marco de referencia del visitante. Estos factores son tan determinantes para captar y mantener la atención como para favorecer los procesos de aprendizaje.

En la literatura y entre los diseñadores de expositores, el éxito de un módulo se establece tanto por su capacidad de atracción, que se mide sumando el número de personas que se detienen frente a él, como por el tiempo de permanencia que esas personas le destinan (Boisvert; Slez, 1994; Koran et al., 1986; Wolf y Timitz, 1978a).

Rosenfeld $(1979,1981)$ sugiere que el poder de mantenimiento de la atención en el caso de los grupos familiares está íntimamente relacionado con la interacción que se desencadena frente al módulo.

Todas estas perspectivas consideran que el tiempo de permanencia en los expositores es un indicador de aprendizaje. Sin embargo, no se observa en la literatura una inquietud por describir de qué aprendizaje se trata (¿receptivo, memorístico, significativo?), ni tampoco se comenta el grado de estabilidad, extensión, generalización o conservación del mismo. En pocas ocasiones se examina con cierto detalle el carácter de este aprendizaje; así, por ejemplo, y como excepción, Falk (1983) encuentra correlaciones significativas entre el tiempo de atención destinado a los módulos y las respuestas de los visitantes a un cuestionario abierto sobre la comprensión conceptual de la exposición.

Las investigaciones mencionadas más arriba invitan a adoptar un uso prudente del término aprendizaje.

\section{ACTIVIDAD COMPARTIDA Y OPORTU- NIDADES DE INTERACCIÓN FRENTE A LOS EXPOSITORES}

Del mismo modo que en la literatura se acepta que el tiempo de atención es un indicador de aprendizaje, la actividad compartida entre miembros de un grupo familiar puede informar sobre la intención educativa que los visitantes incluyen en sus «agendas». El concepto de agendas no remite sólo a la idea de plan de visita o ejecución de la misma, sino al conjunto de expectativas que se generan al decidir visitar un centro de ciencia. En los últimos años se han realizado numerosos estudios (McManus, 1994) sobre la combinación de las «agendas placenteras y educativas» que los adultos emplean para dar soporte a los aprendizajes de sus hijos (Hilke, 1989). En este sentido se reconoce que la diversión no sólo procede de la manipulación de los módulos sino también de poner en funcionamiento una íntima unidad social en un lugar público elegido libremente. Desde esta perspectiva tiene sentido examinar las conductas compartidas entre los diferentes miembros de las familias.

Diamon (1980-86) caracteriza algunos procesos de enseñanza espontáneos que ocurren en la interacción familiar y que tienen lugar gracias a una actividad recíproca entre diferentes miembros de la familia: mientras los niños tienden a compartir entre ellos información concreta, por ejemplo, sobre las operaciones que ejecutan los módulos, los adultos tienden a compartir información simbólica recogida a través de la lectura de carteles o de su conocimiento y experiencia previa.

Taylor (1986), estudiando a familias en el Acuario de San Francisco, observa que hablan de lo que conocen. Discuten sobre el expositor en términos de su recuerdo y conocimiento previo.

Estas discusiones refuerzan la experiencia pasada y la historia familiar y contribuyen a construir una comprensión compartida entre los miembros de la familia. De este modo, la familia provee a los niños de conexiones entre lo que exhiben los museos y el conocimiento y experiencias anteriores.

Parece que la formación de las personas que visitan los museos constituye una variable decisiva sobre los aprendizajes que puedan tener lugar. Taylor (1986) señala la importancia del conocimiento previo en las nuevas adquisiciones. Shettel y otros (1979) observan que, cuando las personas van a una exposición sabiendo ciencia, tienden a aprender más que los que no saben.

Estos datos permiten suponer que la formación de los progenitores juega un papel determinante en los aprendizajes de la familia.

La mayoría de trabajos que hemos citado, o bien examinan algunas características de la población en relación con ciertas conductas de aprendizaje, o bien examinan conductas interactivas entre los miembros de la familia $\mathrm{y}$ entre ellos y los expositores.

Muchos de los trabajos descritos más arriba se interesan por los aprendizajes que tienen lugar en las visitas al museo. Sin embargo estas investigaciones suelen poner el foco, o bien en las características demográficas de los visitante, tomándolas como variables independientes, o bien en las conductas de aprendizaje propiamente dichas. Los resultados obtenidos informan sobre el uso que se hace del museo y también del éxito de los expositores, pero dicen muy poco sobre las condiciones del aprendizaje efectivo y las variaciones de la influencia educativa que los adultos ejercen sobre los más pequeños. Mejorar la comprensión sobre esta influencia probablemente requiera algo más que un análisis cuantitativo; sin embargo, creemos que cuantificar y cruzar variables sigue siendo útil para conocer qué factores de la influencia educativa intervienen en la interacción. 


\section{LA INFLUENCIA EDUCATIVA DE LOS PADRES FRENTE AL «MODULO DE LAS OLAS»}

Este trabajo se propone diferenciar la influencia educativa de la actividad entre progenitores de diferente género. Nos preguntamos si se comportan del mismo modo las mamás y los papás o difieren en alguna actividad con sus hijos/as. Así mismo la formación de los progenitores tal vez influya en el tipo y grado de la interacción con los niños. Trataremos de averiguar también si hay diferencia en la actividad compartida entre los progenitores que poseen una formación académica (agrupando indistintamente los que han cursado carreras científicas o humanísticas) y quienes no poseen un título universitario. También se comparará los que poseen una formación científica y los que poseen otro tipo de formación.

Nuestro estudio tendrá en cuenta dos variables demográficas de una muestra de visitantes: la formación y el género, con relación a cinco actividades que pueden ser compartidas con los niños. Éstas son: la atención, el interés, la manipulación del módulo, el diálogo entre niños y adultos y la lectura de textos.

La relación entre ambos tipos de variables tal vez permita conocer mejor cómo afectan los aprendizajes algunas condiciones de las familias. Concretamente saber cómo las dos variables demográficas influyen en la actividad conjunta entre una díada permitirá obtener criterios para seleccionar aquellas muestras más representativas, para continuar profundizando en estudios posteriores.

\section{METODOLOGÍA}

\section{Sujetos}

La muestra fue seleccionada después de una grabación en vídeo frente al módulo y de una entrevista en la que se preguntaba por la relación de parentesco entre los miembros del grupo que acababa de ser grabado. Los sujetos de esta investigación son díadas: padre/madre, hijo/a. El número de parejas analizadas fue en total de 85 , de las cuales 46 eran hombres; 39 , mujeres; 40 , niños y 45 , niñas.

\section{Técnica}

Se colgó una cámara de vídeo y un micrófono en el techo enfocando «La máquina de las olas». Estos aparatos más un monitor de televisión permanecieron ocultos. El vídeo se pone en marcha cuando algún miembro del grupo familiar entra en escena y se detiene cuando los dos miembros (niño/a y adulto/a) salen del foco. Dos personas están frente al monitor, una sale al encuentro de la familia, al finalizar la grabación, para entrevistarla y pedir su consentimiento para conservar las imágenes.

\section{Tarea y contexto}

Se grabaron solamente aquellas familias que se detenían delante del expositor durante un tiempo superior a 30 segundos. El expositor se encuentra en la sala «El planeta vivo». Su ubicación constituye el inicio del más probable de los recorridos. Las familias que se detienen en ese módulo presumiblemente inician su visita al museo a partir de este punto, lo que favorece su interés y atención.

«La máquina de las olas» consiste en un receptáculo rectangular lleno de agua. El agua alcanza dos terceras partes de todo el volumen. Hundida en ella y en uno de sus extremos hay una palanca accionable a través de un dispositivo exterior a cuya presión responde produciendo un movimiento del agua que simula las olas. En el otro extremo se encuentra una montañita de piedras, y a dos aguas hay una pelota. Cerca de la palanca y colocado en una superficie perpendicular a ella se encuentra el texto explicativo.

La intención del expositor es mostrar que el movimiento de las olas es horizontal sólo aparentemente. Cuando la palanca se activa, se observa el movimiento de la pelota en sentido vertical. Esto permite suponer que el agua no se desplaza sino que se mueve verticalmente.

Así mismo, en la base del módulo se encuentra una fina capa de arena en toda la superficie cuya forma alude al movimiento del agua pero poniendo de manifiesto que no hay desplazamiento.

Este expositor reúne todas las características de idoneidad para mantener la atención de las familias: presenta un desafío a las ideas implícitas sobre el sentido del movimiento del agua; contiene elementos familiares y de fuerte poder simbólico como son las olas, arena, playa...; permite una inferencia a través de una actividad reflexiva sobre el movimiento de la pelota; $y$, finalmente su gran volumen contribuye a realizar una observación cómoda para los grupos de visitantes.

\section{Procedimiento}

El corpus del trabajo agrupa las entrevistas realizadas a 85 familias y las respectivas transcripciones de los vídeos. Cada una de éstas se ordenan en un protocolo cuyas columnas contienen lo que hacen y dicen los participantes y las filas, quién es el sujeto de cada evento: el progenitor o el niño.

\begin{abstract}
ANÁLISIS
Se realizó un análisis cuantitativo no paramétrico con la intención de probar el carácter discriminatorio de las categorías de análisis sobre la conducta y las variables demográficas. El objetivo último fue afinar un instrumento que permitiera posteriormente hacer un análisis cualitativo sobre algunos casos elegidos.
\end{abstract}


Fueron codificadas las respuestas de la entrevista así como algunas actividades y conductas registradas en los vídeos.

Las variables y categorías que se tomaron en cuenta para elaborar la base de datos fueron las siguientes:

\section{Categorías de análisis}

Datos de la entrevista

1. Género del progenitor.

2. Género del niño.

3. Profesión del progenitor (1. Carreras universitarias de ciencias. 2. Carreras universitarias de letras. 3. Carreras medias. 4. Amas de casa. 5. Profesiones no cualificadas (listas en el anexo I).

4. Edad del niño/a (entre 9 meses y 13 años).

5. Miembros de la familia que acompañan a la díada (1. Sin familia. 2. Con otros adultos. 3. Con otros niños. 4. Con otros adultos y otros niños).

\section{Datos de la conducta}

6. Atención. 1. Atención conjunta y simultánea: la díada llega junta al módulo. 2. Conjunta y alternada: ambos llegan juntos pero luego se separan. 3 . No conjunta y no hay llamada: no llegan juntos y ninguno llama al otro. 4. Llegan separados pero el progenitor llama al niño. 5. Llegan separados pero el niño llama al progenitor.

7. Desinterés. 1. Conjunto: abandonan el módulo a la vez. 2. El niño se va antes. 3 . El progenitor se va antes.

8. Activación del módulo. 1. El módulo ya está activado. 2. Activa el padre. 3. Activa el niño. 4. El padre propone al niño que active.

9. Diálogo entre la díada. 1. Cuando hay más de cuatro emisiones, entre ambos se considera que hay diálogo. 2. Cuando hay menos de cuatro emisiones se considera que no lo hay.

10. Lectura del progenitor. 1. Lee en silencio. 2. Lee en voz alta. 3. No lee.

11. Lectura del niño. 1. Lee en silencio. 2. Lee en voz alta. 3. No lee.

\section{Tratamiento estadístico}

El tratamiento estadístico de los datos se realizó empleando el programa SPSS. Se hizo un análisis de frecuencias para todas las categorías de cada variable y un análisis de significaciones $(\chi 2)$ entre parejas de variables.

\section{RESULTADOS}

\section{Frecuencia de cada variable y descripción de la muestra}

La muestra de progenitores (Fig. 1) se reparten entre quienes poseen carreras universitarias largas y cortas, los estudiantes y las amas de casa y aquéllos que ejercen oficios.

Figura 1

Formación de los progenitores.

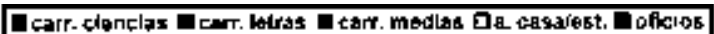

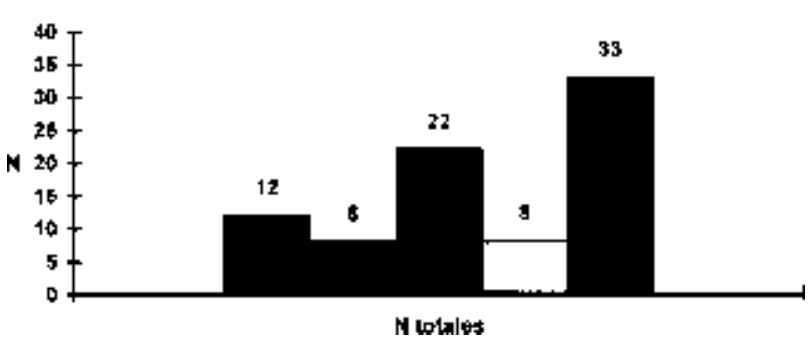

Los padres y las madres vienen acompañados mayoritariamente de niños entre los 4 y los 10 años. Habitualmente se trata de una díada compuesta por un adulto y un niño.

La mayoría de parejas llegan juntas al módulo y lo abandonan juntas. Cuando el expositor no está activado, habitualmente lo activa el niño, por iniciativa propia o por indicación del adulto. La mitad de las díadas mantiene un diálogo frente al expositor, el resto permanece casi en silencio.

La mayoría de padres e hijos no leen los textos y muy pocos lo hacen en voz alta.

\section{GÉNERO DE LOS PROGENITORES EN LA ACTIVIDAD COMPARTIDA}

La variable género de los progenitores no influye de modo significativo en ninguna de las conductas analizadas.

De los cruces entre ésta variable demográfica y la actividad compartida destacan algunas diferencias sensibles cuyas implicaciones interactivas parecen interesantes. Por ejemplo, parece que la relación entre el diálogo y no diálogo (Fig. 2) que mantienen los progenitores con sus hijos se invierte entre los papás y las mamás.

Mientras la mayoría de papás dialoga con sus hijos, la mayoría de mamás no lo hace. Así mismo, aunque la mayoría de la muestra se inclina por no leer, parece que las mamás superan en un $10 \%$ la lectura silenciosa de los papás (Fig. 3). 
Figura 2

Género pad. / diálogo.

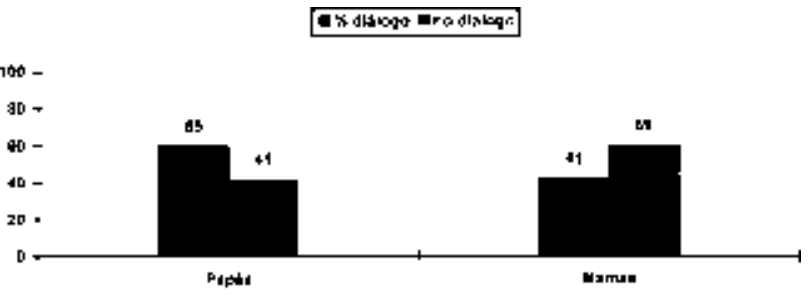

Las variables «Atención y desinterés» no parecen sensibles a las diferencias entre progenitores. Ambos coinciden en el mismo patrón. Cuando el abandono del módulo no es conjunto, quien primero lo deja son los progenitores mientras que los niños permanecen observando en solitario un rato más. Por otra parte, parece que más niños abandonan el módulo solos cuando van con el padre que cuando van con la madre.

\section{LA FORMACIÓN DE LOS PROGENITORES EN LA ACTIVIDAD COMPARTIDA}

La formación de los progenitores influye de modo significativo en la presencia de diálogo con sus hijos $(\chi 2=7,547 ; \mathrm{DF}=2 ; \mathrm{P}=0,002$. Frecuencia esperada $=9,880)$. Éste es el único resultado significativo de este estudio. Para encontrarlo ha sido preciso reagrupar la población del siguiente modo (Fig. 4): los que ejercen carreras universitarias de ciencias y letras, los que ejercen carreras medias y los que realizan oficios junto con las amas de casa.

Este resultado indica que es más probable que los padres que dialogan con sus hijos se encuentren entre los que poseen una carrera universitaria que entre los demás.

Adoptando esta misma agrupación de la muestra se observa que la formación de los padres arroja diferencias sensibles aunque no significativas en otras conductas. Por ejemplo, los que poseen una formación no universitaria y los que ejercen oficios leen más que quienes poseen una formación universitaria (Fig. 5). Aunque la

Figura 3

Género pad. / lectura.

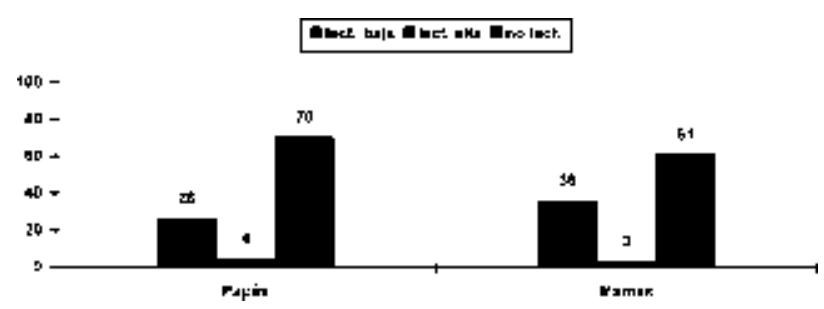

456
Figura 4

Formación agrup. / Diálogo.

ai didage Dno daílapo

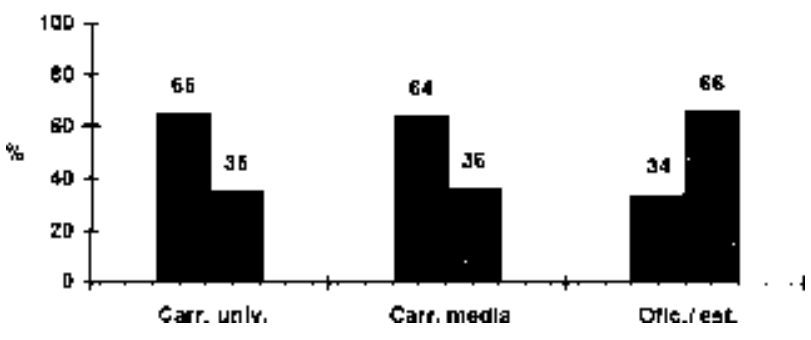

mayoría de la muestra no lee, son los universitarios quienes recurren a la lectura en voz alta. De este modo comparten la información con sus hijos.

Por otra parte, la mayoría de progenitores dirige su atención sobre el expositor de forma conjunta con sus hijos. Si agrupamos las categorías de atención conjunta (1 y 2) y las restantes de atención no conjunta $(3,4$ y 5$)$, se observa que en la primera agrupación son las amas de casa y los que ejercen oficios quienes presentan una atención conjunta mayor.

En cuanto al abandono del módulo, la mayoría lo hace conjuntamente. Las deserciones en solitario corresponden a los progenitores que ejercen oficios y a las amas de casa, en primer lugar, seguidos por los que poseen carreras medias. Los universitarios no parecen tan inclinados a cambiar el foco de su atención de forma unilateral.

Por último, la variable formación presenta cierto contraste entre aquéllos que activan el módulo y quienes proponen a los niños que lo hagan. Aunque muchas familias se encuentran con el módulo ya activado, hay 18 adultos que ponen en marcha la máquina. Entre ellos no hay ningún universitario.

Figura 5

Lectura padres / Formación agrup.

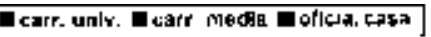

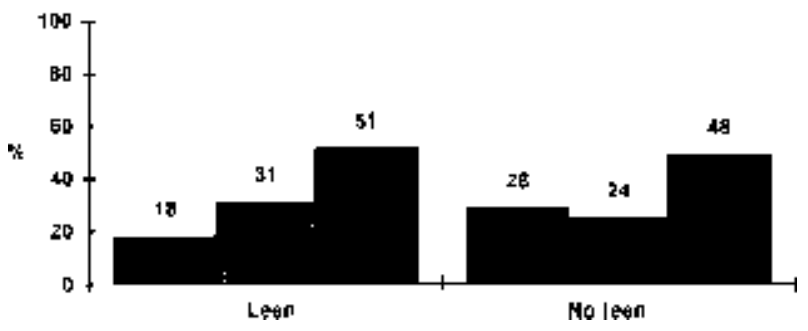

ENSEÑANZA DE LAS CIENCIAS, 1998, 16 (3) 
No se han encontrado diferencias significativas entre quienes poseen una carrera científica y el resto de la población. Las categorías de análisis elegidas para este estudio no permiten discriminarlos.

\section{APRENDIZAJE COMPARTIDO, ENTRE PADRES E HIJOS FRENTE AL MÓDULO}

Los diálogos entre padres e hijos merecen un análisis detallado que no es posible incluir aquí en profundidad. Sin embargo, podemos decir que en ellos se ponen de manifiesto secuencias acción -evento de patrones comunicativos bastante comunes entre las familias sea cual sea la formación de los padres y su género.

En aquellas secuencias en que los miembros de las familias tratan de sacar información del módulo y la intercambian con sus miembros, habitualmente se encuentran pautas de pregunta/respuesta con una variedad de intenciones como: verificar, denegar, describir, interpretar, informar, entre otras (Hilke, 1989).

Más allá de estas secuencias, los diálogos que los padres establecen con sus hijos sobre el fenómeno científico exhibido ponen de manifiesto a menudo el proceso comprensivo de aquéllos. En ocasiones es muy clara la premisa «quien enseña aprende»; en otros, se puede ver cómo los padres modulan e interpretan la información empleando tanto los medios que ofrece el módulo como su rico bagaje de recuerdos y experiencias anteriores.

Ofrecemos dos diálogos que revelan un proceso comprensivo entre padres e hijos sobre el módulo de las olas.

Una madre y su hija de 4 años llegan juntas. La madre se coloca frente al texto explicativo. Lee en voz alta mientras la niña está a su lado esperando. A continuación se dirige al módulo lleno de agua, a la altura donde se encuentra la pelota a dos aguas.

Mamá:-Las olas parecen moverse hacia la playa... se rompen; sin embargo, la pelota... iMira la pelota! (Señala la pelota con la mano.) Está alrededor de las olas... siempre en un mismo punto, se ve además la arena del fondo y tampoco avanza. (Ambas, madre e hija, se desplazan juntas hacia la parte que simula una playa.)

Mamá: -Las olas... Mira, el agua que parece que va a la playa, ¿no? ¡Mira! Y aquello es la playa, ¿no? Sin embargo, la pelota está siempre en el mismo sitio... ¿o no? (Ambas miran atentamente y en silencio los movimientos del agua y la pelota.)

Niña, señalando con el dedo: -Pero un poco se mueve.

Mamá: -Yo creo que sí. (Inmediatamente se desplaza hasta las instrucciones, que lee en silencio. Vuelve a mirar la pelota en silencio.)
Niña, señalando la pelota con el dedo: -iSí, mira, mami, mira!

Mamá:-Tiene que ser así; lo que pasa es que tiene que ser así. Lo que pasa es que va dando vueltecitas... (Sigue los círculos imaginarios del agua con el dedo.) Hace así, ¿ves? (Recorre el contorno de la ola con el dedo.) No se mueve por aquí, por encima de la ola, no. Va dando vueltecitas como el dibujo que hace aquí. (Desplazándose hacia las instrucciones señala con el dedo el dibujo encajado en el texto.) Mira aquí. ¿Ves? La pelotita. Va dando vueltas.

Un padre y su niño de 7 años llegan juntos y se colocan frente al módulo, que el padre se encarga de activar.

Papá: -iMira! Esto hace olas. (Miran en silencio ambos.)

Papá: -Mira la pelota; tu, mira la pelota. Ya está, ya está. Ves, esto son las olas. (Las señala con la mano mientras ambos se desplazan hacia el lugar donde está la pelota.)

Papá, mirando al niño y después a la pelota: $-Y a$ verás cuando llegue aquí a la costa (al otro extremo del módulo). (Se queda mirando la pelota con los brazos cruzados.)

Niño, delante del padre y mirando la pelota: - ¡No llega!

Papá: -Ya va ha llegar, ya... (Miran juntos la pelota que permanece en el mismo lugar.)

Niño:-Ahora... (Mientras hace rotaciones con el dedo siguiendo el movimiento de la pelota.)

Papá:-Las olas del mar. El viento; en este caso, sería el viento... (Dibuja en el aire la forma sinuosa de la ola.)

Niño: -Mira ésta. (Señala con el dedo la ola e imita las olas con el brazo.)

Papá, señalando la pelota con el dedo:-¿Ves? De aquí ya no se mueve más.

Niño:-¿ ¿Por qué? ¿Por qué tiene esto?

Papá: -El agua ha de subir hacia arriba, esta agua de aquí empuja hacia allí. (Hace gestos con la mano en sentido vertical.)

Algunos diálogos ponen de manifiesto diferencias en la selección de la información entre los niños y los adultos. En el ejemplo siguiente la niña (10 años) quiere compartir con su madre el deseo de conseguir una meta. Para ello trata de centrar la atención de la madre en la altura que presumiblemente alcanzaran las olas en el módulo. Por su parte la madre atiende pero señala otro dato que le llama más la atención: la posición de la pelota a pesar del movimiento del agua.

Niña: -iMira esto! 
La mamá va al lado de la niña y mira atentamente como ella está apunto de apretar el botón que activa la palanca. Finalmente lo aprieta.

Niña: -Pero mira. ¿Ves? Ya verás. ¡Mira ahora, ya verás! Espera un poquito y mira cómo es el agua. (Mira atentamente cómo aumenta progresivamente la altura de las olas... parece esperar algo espectacular.)

Mamá:-Las olas.

Niña:-...Sí, pero quedan levantadas. (Señalando con el dedo las olas. Las miran conjuntamente.)

Mamá: - iMira la pelota!

Niña: -Ya, pero es que antes aún subían más alto. (Se va hacia el lado de la playa.)

Mamá:-La pelota sigue aquí... ¿Ves la pelota?

Niña, caminando en dirección al movimiento de las olas y siguiendo con el dedo: -Me gustaría ser pequeña y nadar por aquí.

Recordamos un caso que nos impresionó mucho por la habilidad con que la madre aprovechó el módulo para enseñar a su hija deficiente de quince años. Esta secuencia lamentablemente no quedó grabada, de forma que tuvimos que transcribirla directamente.

La madre, frente al módulo y mostrando a la chica los movimientos de sube y baja de la pelota, tendía un puente entre una experiencia anterior y la información del módulo. Explicaba a su hija que esos movimientos de la pelota eran los mimos movimientos que ella hacía al flotar en el mar durante el verano.

Mamá:-Fíjate por qué te digo que debes tener cuidado en no irte muy lejos de la playa. Porque el agua no te traerá a la playa igual que no lo hace con esta pelota. Se mueve arriba y abajo pero no se desplaza.

Estos ejemplos dan idea de la versatilidad y recursos de los aprendizajes en familia. El grupo familiar puede funcionar como un sensible y flexible sistema de aprendizaje. No sólo en el ámbito de la vida cotidiana, cuyo escenario le ha pertenecido siempre, sino también en otros escenarios cuyas características están aún poco definidas desde una perspectiva sociocultural.

\section{DISCUSIÓN}

Hay evidencias de que el museo ejerce de instrumento educativo para las familias que lo visitan durante el fin de semana. Una serie de indicadores permiten suponer que su influencia repercute en la actividad conjunta y la interacción con los niños.

Con independencia del género y formación de los padres se observa una actitud generalizada de acompañamiento y soporte frente a la experiencia común que les ofrece «La máquina de las olas». Estos progenitores animan a los niños a actuar sobre la máquina, observan cómo lo hacen, les ayudan en sus requerimientos, escuchan y responden a sus comentarios, les acompañan durante la observación e interacción con el módulo y les permiten seguir observando aunque para ellos el interés se haya agotado.

El soporte que los padres ofrecen a la actividad de los niños facilita y hace más probable la adquisición de experiencias y conocimientos.

De esta actitud generalizada, sin embargo, destaca la influencia de la formación de los adultos en la esfera de la comunicación verbal que abarca el diálogo y la lectura en voz alta.

Hay más comunicación verbal entre aquellas díadas cuyo progenitor posee una carrera universitaria. Este dato sugiere muchas interpretaciones pero sobre todo indica la conveniencia de ampliar y orientar los estudios sobre la influencia educativa de las familias en la dirección de un análisis del contenido comunicativo. Una aproximación cualitativa permitiría conocer los mecanismos de aprendizaje que subyacen a la conducta descrita en este estudio. No se han encontrado diferencias importantes entre progenitores de diferente género; también aquí un análisis cualitativo ofrecería más información.

Por otra parte, los resultados de la lectura de carteles y textos podrían tener consecuencias sobre el diseño de los expositores. A pesar de que la mayoría de las personas no leen, es interesante conocer qué formación poseen los lectores que se benefician de los textos. En nuestro estudio recurren a la lectura aquéllos que no poseen una formación universitaria. Tal vez estas personas confían más en comprender la experiencia a partir de la lectura que exclusivamente accionando el módulo. También este punto merece un análisis más detenido. Para adaptar el contenido de los textos al público no parece suficiente conocer quiénes los usan y por qué. Sería conveniente además averiguar qué hacen con la información escrita.

Los estudios de familia permiten registrar el uso de los conocimientos en una dinámica de interacción social. Los comentarios que hacen los padres a sus hijos, las respuestas a las preguntas de los niños, la secuencia de conductas entre lo que se hace y lo que se dice, todo ello configura una fuente de información muy rica y útil para mejorar los expositores y conocer cómo funcionan estos aprendizajes en contextos no formales. 


\section{AGRADECIMIENTOS}

Agradecemos el interés prestado por el Museo de la Ciencia de la Fundación «La Caixa» de Barcelona; muy especialmente a Jorge Wagensberg, quien nos abrió sus puertas; a Eva Villuendas y Mercedes Lojo, que colaboraron en la recogida de los datos, y a Jordi Vives y María Prats, que facilitaron el registro de los mismos.

\section{REFERENCIAS BIBLIOGRÁFICAS}

AUSUBEL, D., NOVAK, J.D. y HANESIAN, H. (1978). Educational Psychology. A cognitive view. 2a. ed. Nueva York: Holt, Rinehart and Winston. Trad. cast. de Sandoval, M. 1983. Psicología educativa. México DF: Trillas.

BOISVERT, D.L. y SLEZ, B.J. (1994). The relationship between visitors characteristics and learning associated behaviors in science museum. Discovery Space. Science Education, 78(2), pp. 137-148.

BULTER, B. y SUSSMAN, M. (1989). Museum visits and activities for family life enrichment. Marriage and Family Review, 13(3), p. 4.

DIAMOND, J. (1980). The ethnology of teaching: A perspective from the observation of families in science centers. Dissertation Abstracts International, 40, 3510A.

DIAMOND, J. (1986). The behavior of family groups in science museums. American Museum of Natural History, 29(2), pp. 136-154.

DIERKING, L. y FALK, J. (1994). Family behavior and learning in informal science settings: A review of the research. Science Education, 78(1), pp. 57-72.

FALK, J. H. (1983). Time and behavior as predictor of learning. Science Educator, 67, pp. 267-276.

FALK, J.H. y DIERKING, L.D. (1992). The museum experience. Washington, DC: Whalesback Books.

GELMAN, R., MASSEY, C. y McMANUS, M. (1991). Characterizing supporting environments for cognitive development: lessons from children in a museum, en Resnik, L., Levin, J., Teasley, S., Socially shared cognition, pp. 226-256. Washington: American Psychological Association.

HILKE, D.D. (1989). The family as a learning system: An observational study of families in museums, en Butler, B. y Sussman, M., Museum visits and activities for family life enrichment. Marriage and Family Review, 13, pp. 3-4.

KORAN, J.J., KORAN, M.L. y LONGINO, S.J. (1986). The relationship of age, sex, attention and holding power with two types of science exhibits. Curator, 29, pp. 227-235.
Agradecemos también a Juan Giménez su asesoramiento técnico. Finalmente nuestra gratitud a Carles Riba y Francesc Martínez por sus sugerencias y orientaciones metodológicas, así como a Carme Tomás, directora de Servicios Pedagógicos del IMEB mientras se realizó este proyecto durante el curso 1995-96.

LAKOTA, R.A. (1975). The national museum of natural history as a behavioral environmental. Unpublished manuscript. Smithsonian Institution, Washington, DC.

McMANUS, P. (1987). It's the company you keep...The social determination of learning-related behavior in a science museum. The International Journal of Museum Management and Curatorship, 6, pp. 263-270.

McMANUS, P. (1994). Families in museums, en Miles, R. y Zavala, L., Towards the museum of the future: new European perspectives. Londres, EEUU, Canadá: Routledge.

ROSENFELD, S. (1981). Informal education in zoos: Naturalistic studies of family groups. Dissertation Abstracts International, $40,3601 \mathrm{~A}$

ROSENFELD, S. (1979). The context of informal learning in zoos. Roundtable Reports, 4, pp. 3-4.

SCRIBNER, S. y COLE, M. (1973). Cognitive consequences of formal and informal education. Science, 132, pp. 553-558. Trad. cast., 1982. Consecuencias cognitivas de la educacion formal e informal. Infancia y Aprendizaje, 7, pp. 3-18.

SHETTEL, M. et al. (1968). Strategies for determining exhibit effectiveness. Pitsburgh: Thecnical Report. AIR-F58-11/67 FR.

SCHOUTEN, F. (1987). Psychology and exhibition design, a note. Museum Management and Curatorship, 6, pp. 259-262.

TAYLOR, S. (1986). Family behavior at the Steinhart Aquarium. Tesis doctoral no publicada. Universidad de California: Berkeley.

VALSINER, J. y LEUNG, M.C. (1994). From intelligence to knowledge construction: a sociogenetic process approach, en Sternberg y Wagner (eds.). Mind in Context. Cambridge: Cambridge University Press

WOLF, R. y TYMITZ, B.L. (1979). Do giraffes ever sit?: A study of visitor perception at the national Zoological Park. Smithsonian Institution, Washington, DC. 


\section{ANEXO I}

\section{FORMACIÓN DE LOS PROGENITORES}

1. CARRERAS UNIVERSITARIAS DE CIENCIAS

Médico

Economista

Matemático

Arquitecto

Óptico

Ingeniero

Químico

Farmacéutica

2. CARRERAS UNIVERSITARIAS DE LETRAS

Historiador

Pedagogo

Psicólogo

Abogado

Filólogo

Filósofo

\section{CARRERAS MEDIAS}

Informática

Aparejador

Profesor de educación física

Maestros

Enfermera

Auxiliar clínica

Ingeniero técnico

Electricista

Electrónico

Técnico urbanístico

4. ESTUDIANTE / AMAS DE CASA

5. OFICIOS

Ordenanza

Camarero

Técnico de teatro

Mecánico

Agente de viajes

Comercio

Empleado de banca

Conductor

Patronista

Quiromasajista

Empleado de fábrica

Albañil

Administrativo 